\title{
Long-Term Effect of Alogliptin on Glycemic Control in Japanese Patients With Type 2 Diabetes: A 3.5-Year Observational Study
}

\author{
Kohzo Takebayashia, b, Tatsuhiko Suzukia Rika Naruse ${ }^{a}$, Kenji Hara ${ }^{a}$, Mariko Suetsugu ${ }^{\text {a }}$, \\ Takafumi Tsuchiya ${ }^{a}$, Toshihiko Inukai ${ }^{a}$
}

\begin{abstract}
Background: The goal of the current study was to investigate the long-term effects (after 3 years or more) of alogliptin on glycemic control in Japanese patients with type 2 diabetes.

Methods: We retrospectively studied the effect of alogliptin on glycemic control in the patients with type 2 diabetes who had participated in our previous 3-month study and who continued to take alogliptin for at least 36 months.

Results: The mean duration of alogliptin treatment was $42.8 \pm 2.2$ months. In all 39 patients, a significant reduction in hemoglobin A1c (HbA1c) levels was noted between the baseline and final visit: $7.8 \pm 0.6 \%$ to $7.2 \pm 1.0 \%(\mathrm{P}=0.0001)$. A significant reduction in $\mathrm{HbAlc}$ levels was found in a subgroup of patients who did not change their anti-diabetic drugs or did decrease the dose of their sulfonylureas (SUs) or did change to a lower strength repaglinide $(n=32)$ : $7.7 \pm 0.6 \%$ to $7.2 \pm 1.0 \%(\mathrm{P}=0.0005)$. A significant decrease in lowdensity lipoprotein cholesterol (LDL-C) levels was observed in all of the patients that had LDL-C levels determined $(\mathrm{P}=0.0406)(\mathrm{n}=37)$, and in a subgroup of patients who had not taken either statins, fibrates, or pioglitazone, or who had taken one or more of these drugs but the doses were not changed during the observation period $(\mathrm{P}=0.0250)$ $(n=27)$.
\end{abstract}

Conclusion: The current study found that alogliptin performed well for glycemic control when evaluated by HbAlc levels in a long-term observation period exceeding 3 years in Japanese patients with type 2 diabetes. Alogliptin may also decrease circulating LDL-C levels with long-term use.

Keywords: Alogliptin; Durability; Type 2 diabetes

Manuscript submitted June 26, 2017, accepted July 10, 2017

aDepartment of Internal Medicine, Dokkyo Medical University Koshigaya Hospital, Koshigaya, Saitama, Japan

${ }^{b}$ Corresponding Author: Kohzo Takebayashi, Department of Internal Medicine, Dokkyo Medical University Koshigaya Hospital, 2-1-50, Minami-Koshigaya, Koshigaya, Saitama 343-8555, Japan. Email: takeb@gmail.plala.or.jp

doi: https://doi.org/10.14740/jocmr3118w

\section{Introduction}

It is suggested that good, long-term glycemic control reduces the progression of diabetic complications in patients with type 2 diabetes [1]. Therefore, the importance of the long-term sustained effect, termed "durability", in the treatment for glycemic control of diabetes should be emphasized. In the ADOPT trial, rosiglitazone (a thiazolidinedione), which improves insulin resistance, had better durability for glycemic control compared with that of sulfonylureas (SUs), which are insulin secretagogues [2]. On the other hand, interestingly, although dipeptidyl peptidase (DPP)-4 inhibitors, which block the degradation of glucagon-like peptide (GLP)-1 (a hormone-stimulating insulin secretion in pancreatic $\beta$ cells) resulting in the elevation of circulating active GLP-1 levels, are also included in the category of insulin secretagogues, it is shown that some DPP4 inhibitors (such as vildagliptin, saxagliptin, and sitagliptin) had good durability for glycemic control in a 2-year study [3-7]. Recently, it was reported that alogliptin (a DPP4 inhibitor) also had a better durability for glycemic control compared with that of glipizide (an SU) in a long-term study (2 years) [8]. At present, however, there are no publications demonstrating the durability for glycemic control of alogliptin beyond 2 years.

We previously performed a 3-month clinical study using alogliptin to treat Japanese patients with type 2 diabetes [9]. To investigate the durability of glycemic control by alogliptin for longer than 2 years, we retrospectively explored its effect on glycemic control in the patients with type 2 diabetes who had participated in our previous study and who continued taking alogliptin for at least 3 years (36 months). Before performing this analysis, we hypothesized that alogliptin would demonstrate good durability for glycemic control for more than 3 years in Japanese patients with type 2 diabetes.

\section{Patients and Methods}

\section{Patients}

Our previous study, named APPLE: study of combination effect of AlogliPtin and lansoPrazoLE on glycemic control 
Table 1. Clinical Features at Baseline in Patients With Type 2 Diabetes

\begin{tabular}{|c|c|}
\hline No. (male/female) & $39(20 / 19)$ \\
\hline Age (years) & $69.3 \pm 8.6$ \\
\hline Mean duration of alogliptin therapy (months) & $42.8 \pm 2.2$ \\
\hline BMI $\left(\mathrm{kg} / \mathrm{m}^{2}\right)$ & $24.4 \pm 3.9$ \\
\hline $\mathrm{FPG}(\mathrm{mg} / \mathrm{dL})$ & $164.6 \pm 34.7$ \\
\hline HbA1c (\%) & $7.8 \pm 0.6$ \\
\hline \multicolumn{2}{|l|}{ Diabetic therapy } \\
\hline S/M/A/SM/SMA/SMAP/SP/SPA/none & $12 / 4 / 1 / 8 / 5 / 2 / 2 / 1 / 4$ \\
\hline Statins & 7 \\
\hline Fibrates & 3 \\
\hline
\end{tabular}

Data are expressed as mean \pm SD. BMl: body mass index; FPG: fasting plasma glucose; HbA1c: hemoglobin A1c; diabetic therapy: the number of the patients with respective diabetic therapies; S: sulfonylurea; M: metformin; A: a glucosidase inhibitor; P: pioglitazone.

in patients with type 2 diabetes, was performed from December 2012 to August 2013. The study was registered as UMIN0000009445. The detailed study protocol, including the inclusion and exclusion criteria, was previously described [9]. In brief, the study was a multicenter, randomized, open-label study, and a total of 89 patients were enrolled and completed the study. Patients who received DPP4 inhibitors during 1 month before the study, who changed the type or dose of all drugs including anti-diabetic drugs during the 1 month before the study, who were treated with either proton pump inhibitors (PPIs) or $\mathrm{H} 2$ receptor blockers of all types, and who were treated with insulin were excluded. The patients were randomly assigned into two groups (alogliptin only group and alogliptin + lansoprazole group (a PPI)) and took the assigned drug(s) for 3 months. At the end of this study, most patients continued taking alogliptin, while the administration of lansoprazole was generally discontinued. The inclusion criteria of the current study were 1) continuous administration of alogliptin at least for 3 years by October 31, 2016 and 2) patients treated only in our hospital. In the total 89 patients enrolled in the APPLE study, the number of the patients treated in our hospital was 58 . In these 58 patients, 19 patients were excluded from the study because of the next reasons. Eighteen patients were excluded because they were not be able to be followed up over 3 years due to hospital change $(n=7)$ and self-interruption of visits ( $n$ $=2$ ), or because they discontinued taking alogliptin within 3 years due to self-interruption of taking medicine $(n=1)$, the change to other DPP4 inhibitors (linagliptin $(n=1)$, teneligliptin $(\mathrm{n}=2)$, and vildagliptin $(\mathrm{n}=2))$, the discontinuation due to good glycemic control (hemoglobin A1c (HbA1c): $7.5 \%$ at baseline to $5.5 \%$ at time of suspension $(n=1))$, the change to pioglitazone because of poor glycemic control (HbA1c: $8.7 \%$ at baseline to $8.7 \%$ at the day of change $(n=1))$ and both the discontinuation of pioglitazone and the change to canagliflozin (HbA1: $7.8 \%$ at baseline to $7.0 \%$ at the day of change $(\mathrm{n}=$ 1)). Mean observation periods in these 18 patients were 14.3 \pm 9.0 months. One patient who continued taking alogliptin more than 3 years was also excluded because of the occasional anemia due to probable gastrointestinal bleeding, which may influence $\mathrm{HbA} 1 \mathrm{c}$ levels (HbA1c: $7.8 \%$ at baseline to $6.3 \%$ at final visit). Consequently, the 39 patients (the 20 patients in the lansoprazole group and the 19 patients in the combination group of the previous study) met the inclusion criteria. The main purpose of the original APPLE study was to compare the effect of alogliptin alone and the combination of alogliptin + lansoprazole on glycemic control in patients with type 2 diabetes, because it has been reported that PPIs might improve glucose metabolism in both animal and clinical studies [10]. However, the results of this study were negative for an effect on glycemic control [9] and furthermore, at 1 year after completion of the study (i.e., discontinued use of lansoprazole in the combination group and continued use of alogliptin in both groups), there was no difference in glycemic control between these groups [11]. Therefore, we concluded that lansoprazole had no effect on glycemic control at least in that study cohort. We included the patients from both groups in the current study to increase the number of patients. Accordingly, a total of 39 patients were evaluated in the current study. The clinical characteristics of these patients are presented at Table 1. The mean duration of alogliptin therapy was $42.8 \pm 2.2$ months, i.e., approximately 3.5 years.

\section{Methods}

The 39 patients who met the inclusion criteria for this study were retrospectively analyzed using the medical records from December 1, 2012 to October 31, 2016 for each patient. The day of the final visit to our hospital was defined as the nearest day to October 31, 2016 in which each patient visited to our hospital. The markers of glycemic control including the levels of plasma glucose (PG) and HbA1c, and levels of serum lipids were evaluated in each patient. During the observation period, SUs were changed to repaglinide (a short-term insulin secretagogue with similar mechanisms of action to that of SUs) in some patients. For the change from SUs to repaglinide, we assumed that $1.5 \mathrm{mg} /$ day of repaglinide was comparable to either $2 \mathrm{mg} /$ day of glimepiride, $80 \mathrm{mg}$ /day of gliclazide, or $2.5 \mathrm{mg} /$ day of glibenclamide based on our study and on other previous studies [12-14].

\section{Homeostasis model assessment-insulin resistance (HOMA- IR) and homeostasis model assessment $\beta$ (HOMA- $\beta$ )}

HOMA-IR was used as an indicator of insulin resistance and was calculated as follows: HOMA-IR = fasting PG (FPG) $(\mathrm{mg} /$ $\mathrm{dL}) \times$ fasting circulating immunoreactive insulin $(\mu \mathrm{U} / \mathrm{mL}) / 405$. HOMA- $\beta$ was used as an indicator of insulin secretion and was calculated as follows: HOMA- $\beta=360 \times$ fasting circulating immunoreactive insulin $(\mu \mathrm{U} / \mathrm{mL}) / \mathrm{FPG}(\mathrm{mg} / \mathrm{dL})-63$.

\section{Ethical considerations}

In the original APPLE study, all subjects provided informed 

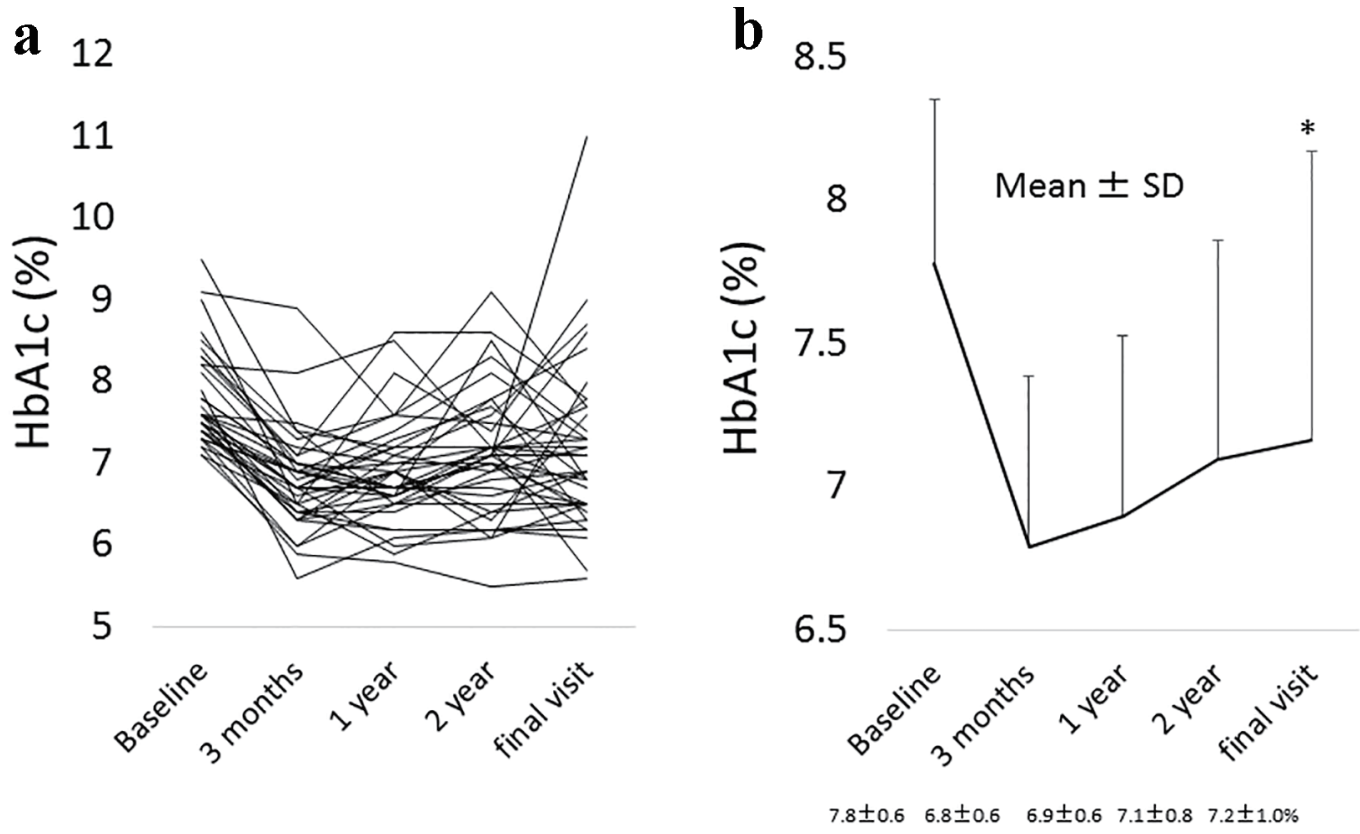

Figure 1. (a) The change in HbA1c levels from the baseline to the final visit with alogliptin therapy in all individuals. (b) The mean change \pm standard deviation (SD) in $\mathrm{HbA} 1 \mathrm{c}$ levels from the baseline to the final visit with alogliptin therapy. *Statistical significance. Final visit: mean 3.5 years from baseline.

consent. The original APPLE study and the current study (analysis) were approved by the Local Ethics Committee in our hospital. The study was performed according to the guidelines of the Declaration of Helsinki.

\section{Statistical analysis}

Comparisons of the two time points for each parameter were performed using a paired $t$-test. Comparisons between the two groups were made using an unpaired $t$-test. All statistical analyses were performed using Ekuseru-Toukei 2012 software (Social Survey Research Information Co., Ltd, Tokyo, Japan). A P value of less than 0.05 was accepted as indicating statistical significance (two-sided).

\section{Results}

In all 39 patients, a significant reduction in HbA1c levels was noted between the baseline and the final visit: $7.8 \pm 0.6 \%$ to $7.2 \pm 1.0 \%$ (95\% confidential interval (CI) for the difference: $0.3-0.9 ; \mathrm{P}=0.0001$ ) (Fig. 1a, b), while plasma glucose did not significantly change during this time frame: $164.6 \pm 34.7$ to $159.1 \pm 46.7 \mathrm{mg} / \mathrm{dL}(95 \% \mathrm{CI}$ for the difference: -7.6 to $18.8 ; \mathrm{P}$ $=0.3992$ ) (Fig. 2a, b). A significant reduction in HbA1c levels was also found in a subgroup of patients who either did not change their anti-diabetic drugs or the SU dose was decreased, or the SUs were changed to a lower strength repaglinide $(\mathrm{n}=$ 32): $7.7 \pm 0.6 \%$ to $7.2 \pm 1.0 \%$ (95\% CI for the difference: 0.3 $0.9 ; \mathrm{P}=0.0005$ ) (Fig. 3). On the other hand, also in the patients who were excluded from the current study because of hospital change, self-interruption of visits or the discontinuation taking alogliptin within 3 years $(n=18)$, there was significant reduction of $\mathrm{HbA} 1 \mathrm{c}$ levels from baseline to the day of final visit or that of the discontinuation of alogliptin: $7.8 \pm 0.6 \%$ to $7.0 \pm 1.0 \%$ (95\% CI for the difference: $0.4-1.2 ; \mathrm{P}=0.0004$ ).

When the patients were divided into two groups based on the median value of either HOMA- $\beta$ or HOMA-IR at baseline (i.e., low and high HOMA- $\beta$ group, and low and high HOMA-IR group, respectively), there was no significant difference in change of $\mathrm{HbA} 1 \mathrm{c}$ levels from the baseline to the final visit between the low and high HOMA- $\beta$ groups $(\mathrm{P}=0.5302)$ and between those in the HOMA-IR groups $(\mathrm{P}=0.4423)$. Furthermore, when the patients were divided into two subgroups based on a body mass index (BMI) value of less than $25(\mathrm{n}=$ 23 ) and that equal to or greater than $25 \mathrm{~kg} / \mathrm{m}^{2}(\mathrm{n}=16)$, significant decreases in $\mathrm{HbAlc}$ levels were obtained in both groups: $7.7 \pm 0.5 \%$ to $7.1 \pm 0.7 \%$ (95\% CI for the difference: $0.3-0.9$; P $=0.0015)$ in the former and $7.9 \pm 0.7 \%$ to $7.2 \pm 1.3 \%(95 \% \mathrm{CI}$ for the difference: $0.1-1.2 ; \mathrm{P}=0.0310)$ in the latter. There was no significant difference in the change of HbA1c levels from the baseline to the final visit between the two groups $(\mathrm{P}$ $=0.8848$ ).

In all patients who had their low-density lipoprotein cholesterol $(\mathrm{LDL}-\mathrm{C})(\mathrm{n}=37)$ and high-density lipoprotein cholesterol (HDL-C) levels $(\mathrm{n}=36)$ determined, there was a significant decrease of LDL-C and HDL-C levels: $106.5 \pm 25.0$ to $96.3 \pm 20.9(95 \% \mathrm{CI}$ for the difference: $0.5-19.9, \mathrm{P}=0.0406)$ for LDL-C, $56.9 \pm 14.9$ to $53.6 \pm 15.1 \mathrm{mg} / \mathrm{dL}$ (95\% CI: 0.02 - 6.54; $\mathrm{P}=0.0488$ ) for HDL-C, respectively (Fig. 4a, b). In a subgroup of patients who did not take anti-hyperlipidemic 


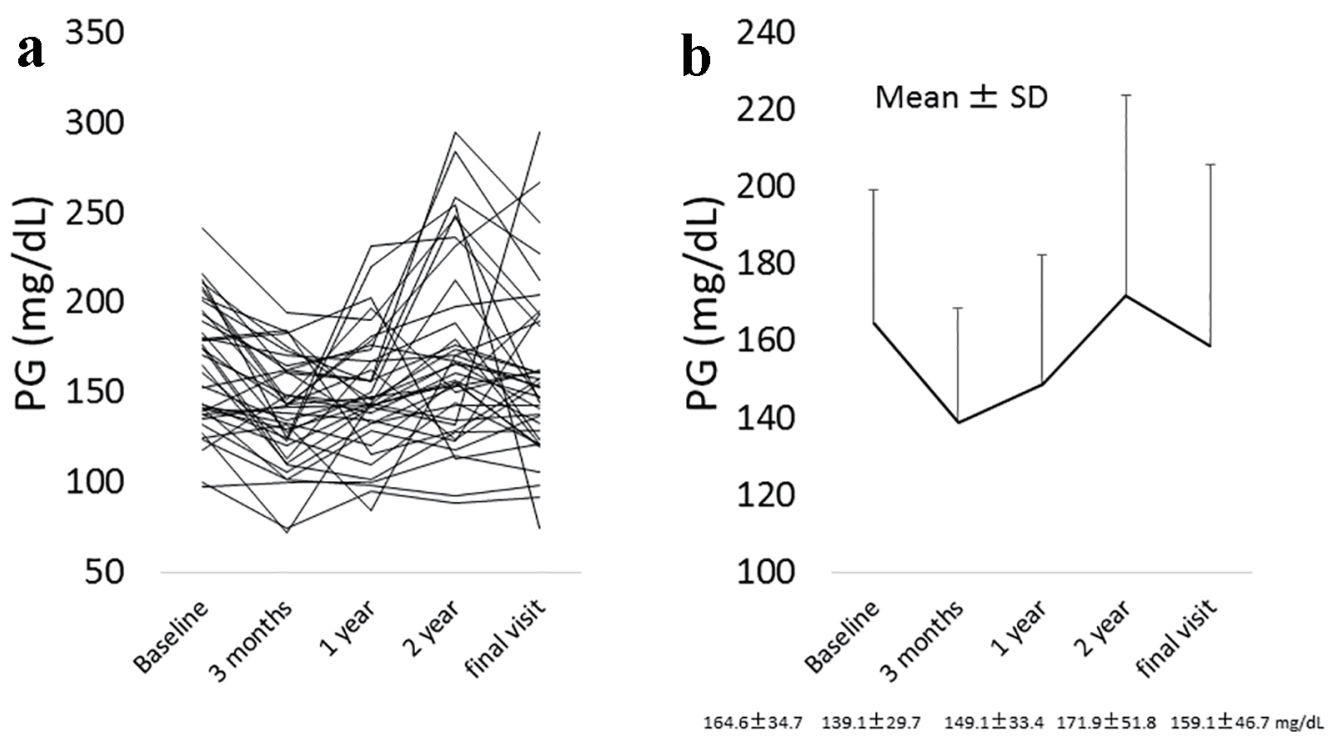

Figure 2. (a) The change in plasma glucose (PG) levels from the baseline to the final visit with alogliptin therapy in all individuals. (b) The mean change \pm SD in PG levels from the baseline to the final visit with alogliptin therapy. Final visit: mean 3.5 years from baseline.

drugs (either statins or fibrates) and pioglitazone (a thiazolidinedione) which can influence serum lipid concentrations based on our and other previous studies $[15,16]$ or who had taken these drugs but the dose was not changed throughout the observation period $(n=27)$, a significant decrease in LDLC levels was maintained: $108.7 \pm 24.9$ to $95.1 \pm 22.8 \mathrm{mg} / \mathrm{dL}$ (95\% CI: 1.8 - 25.3; P = 0.0250) (Fig. 4c). In contrast, a significant decrease in HDL-C levels with alogliptin therapy was not maintained in this subgroup $(\mathrm{n}=26): 56.7 \pm 15.4$ to $54.0 \pm$ $15.1 \mathrm{mg} / \mathrm{dL}$ (95\% CI: -0.7 to $6.3 ; \mathrm{P}=0.1075)$ (Fig. $4 \mathrm{~d}$ ).

\section{Discussion}

In the current study, alogliptin treatment over a mean period of 3.5 years significantly decreased $\mathrm{HbA} 1 \mathrm{c}$ levels by approximately $0.6 \%$ in Japanese patients with type 2 diabetes although a tendency for a small rebound in $\mathrm{HbAlc}$ levels from baseline was noted. This finding was similar even in a subgroup of patients who either maintained their dose of anti-diabetic drugs or decreased their dose of SUs, or changed their SUs to a lower strength repaglinide (approximately $0.5 \%$ reduction). It is reported that a $0.72 \%$ reduction in $\mathrm{HbA} 1 \mathrm{c}$ levels with alogliptin add-on therapy for metformin for 2 years was observed in patients with type 2 diabetes [8]. Although some of the patients in the current study had received multiple anti-diabetic drugs and although the patients' background, such as ethnicity or BMI was different between our study [9] and the previous study [8], our results suggest that the good durability for glycemic control with alogliptin reported in the previous study [8] can be maintained for a longer term exceeding 3 years. The reason for the potentially good durability of alogliptin is unclear. It is possible that the potential protective effect of a DPP4 inhibitor on pancreatic $\beta$ cells as shown in a rodent model of type 2 diabetes [17] was involved although the protective effect of DPP4 inhibitors is not yet determined in humans.

It will be interesting to investigate whether the factors,

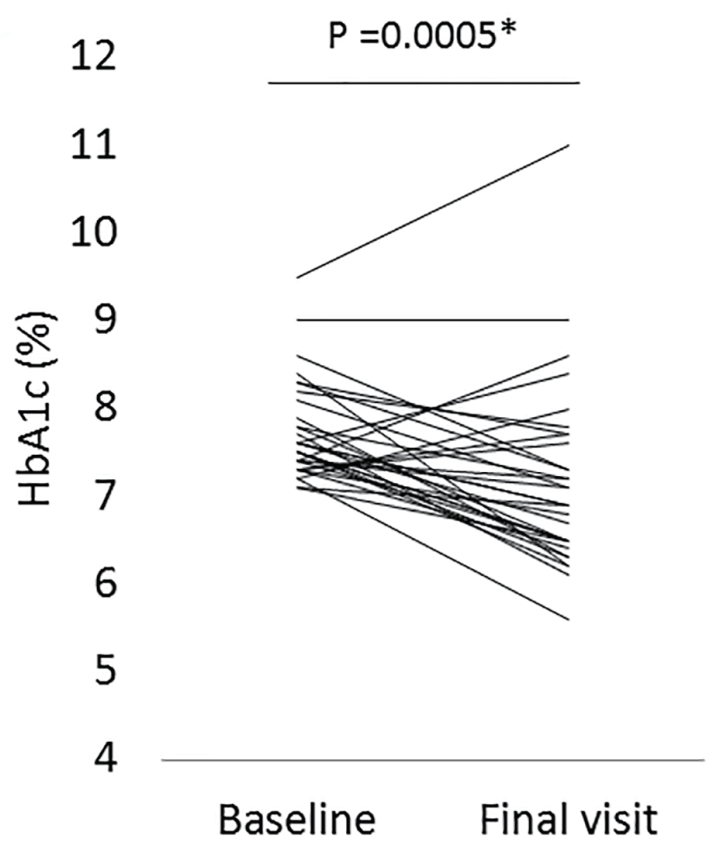

Figure 3. The change in $\mathrm{HbA} 1 \mathrm{c}$ levels from the baseline to the final visit with alogliptin therapy in a subgroup patients who either did not change all of their anti-diabetic drugs or the dose of sulfonylureas (SUs) was decreased, or the SUs were changed to a lower strength repaglinide (n = 32). *Statistical significance. Final visit: mean 3.5 years from baseline. 

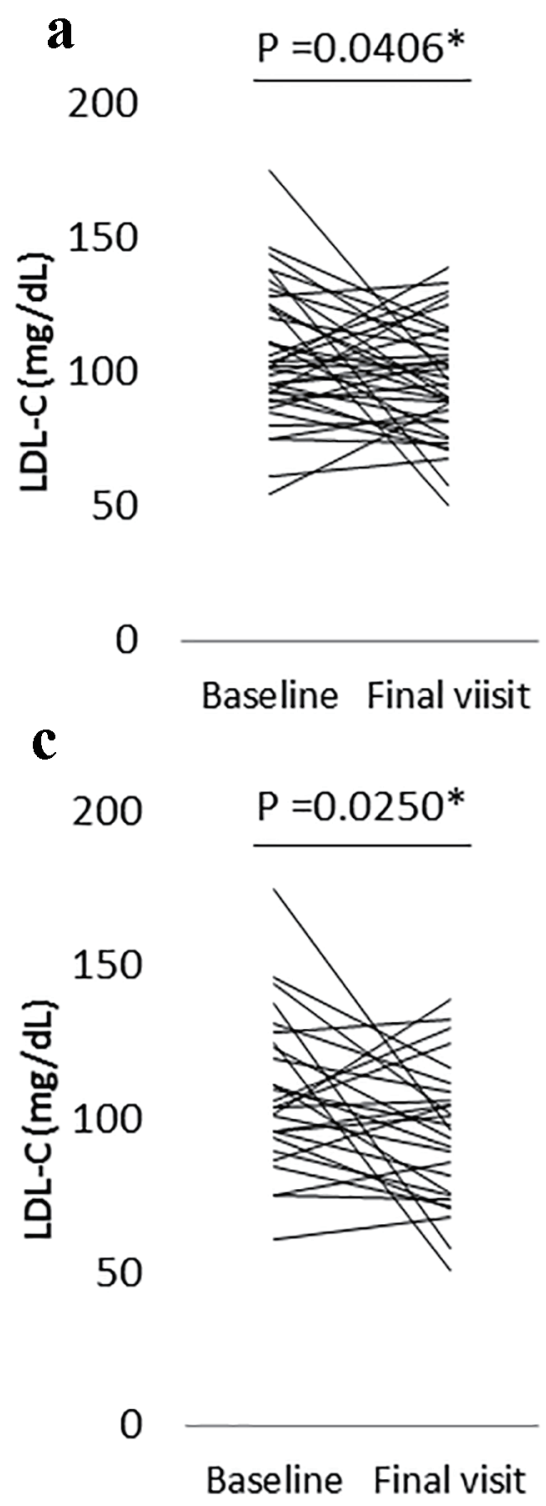
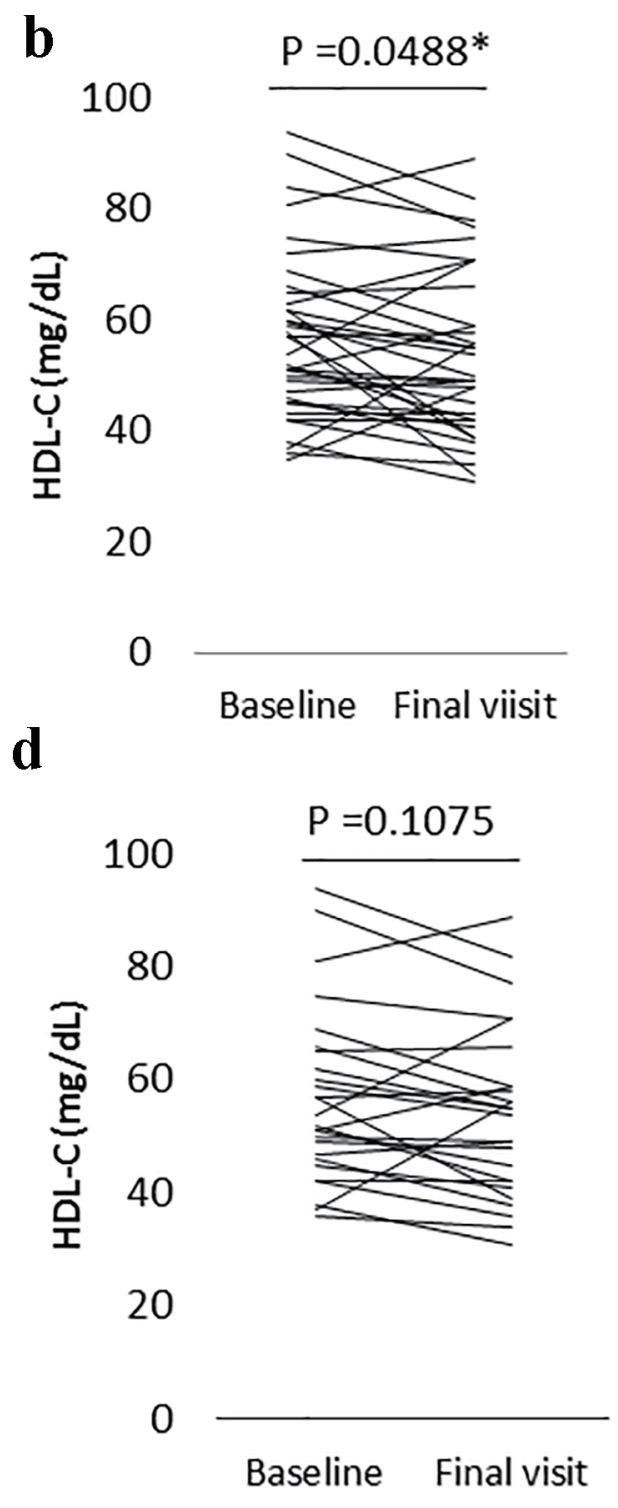

Figure 4. (a) The change in low-density lipoprotein cholesterol (LDL-C) levels from the baseline to the final visit with alogliptin therapy in all patients who had LDL-C levels measured $(n=37)$. (b) The change of high-density lipoprotein cholesterol (HDL-C) levels from the baseline to the final visit with alogliptin therapy in all patients who had HDL-C levels measured $(n=36)$. (c) The change in LDL-C levels from the baseline to the final visit with alogliptin therapy in a subgroup patients who did not take either statins, fibrates, or pioglitazone, or who had taken one or more of these drugs but the dose was not changed during the observation period $(n=27)$. (d) The change in HDL-C levels from the baseline to the final visit with alogliptin therapy in a subgroup patients who did not take either statins, fibrates or pioglitazone, or who had taken one or more of these drugs but the dose was not changed during the observation period $(n=26)$. *Statistical significance. Final visit: mean 3.5 years from baseline.

such as insulin secretion activity and insulin resistance at baseline, can predict the durability for glycemic control with alogliptin or not. We expected that a relatively high HOMA- $\beta$ (an index of insulin secretory activity) and low HOMA-IR (an index of insulin resistance) at baseline may be associated with the good durability of alogliptin because it is likely that pancreatic $\beta$ cell function and insulin resistance influence the clinical effect of alogliptin. However, contrary to our expectation, there was no significant difference in change of $\mathrm{HbAlc}$ from baseline to final visit between the patient subgroups with low and high values of either HOMA- $\beta$ or HOMA-IR. The reason for these negative results is unclear. However, it is possible that the concomitant drugs, such as SUs, metformin, and pioglitazone, influenced the results, and therefore these associations should be investigated in drug-native patients in a future study. In addition, there was no difference between the patient subgroups with low and high BMI which may be associated with circulating DPP4 levels [18], and which therefore may influ- 
ence the effect of alogliptin. However, it is important to note that the mean BMI in this study was not very high (approximately $24 \mathrm{~kg} / \mathrm{m}^{2}$ ), and this may have influenced the result at least in part. On the other hand, in the current study, there was no significant change in PG during the 3.5-year observation period of the study. We suggest that this is due to occasional PG measurements, excluding baseline and 3 months at which time the FPG was evaluated.

In our previous study [9], a tendency toward a decrease in LDL-C levels was noted after 3 months with alogliptin therapy and in the current study, a similar significant decrease in LDL$\mathrm{C}$ levels was found after 3.5 years with alogliptin therapy. The significant decrease was retained also in a subgroup of patients who either did not take drugs which can influence serum lipid levels or who had taken these drugs but the dose was not changed during the observation period. There are reports that in relative short-term studies DPP4 inhibitors, such as alogliptin in 6 weeks [19], and anagliptin in 6 months [20], decreased LDL-C levels, although these findings were not confirmed in other studies [21, 22]. Our current study suggests the possibility that this potential lowering of LDL-C levels by some DPP4 inhibitors, including alogliptin, may be maintained also for a longer term exceeding 3 years. The detailed mechanisms of this clinical LDL-C-lowering effect by some DPP4 inhibitors are still unclear although a very recent report showed that anagliptin may decrease circulating LDL-C levels via the down-regulation of lipid synthesis in the liver in a hyperlipidemic animal model [23]. On the other hand, it is reported that alogliptin also decreased HDL-C levels in a short-term study [19]. However, in the current study, although a significant decrease in HDL-C levels was found with alogliptin treatment after a mean 3.5 years, the significance was not observed in the above-mentioned subgroup. Thus, the robust effect in longterm use of alogliptin on HDL-C levels is still unclear.

The limitations of the current study are that it was a singlearm retrospective observation, and the number of patients was relatively small. The strength of this study was that the patient selection at baseline in the original study was based on robust inclusion and exclusion criteria. Furthermore, the circulating fasting insulin and glucose levels at baseline were measured, allowing for the calculation of HOMA- $\beta$ and HOMA-IR as indicators of insulin secretion and insulin resistance.

In conclusion, we showed in the current study that alogliptin has good durability for glycemic control as evaluated by HbA1c levels in long-term observation periods exceeding 3 years in Japanese patients with type 2 diabetes. Alogliptin significantly decreased LDL-C levels during this observation period in a patient subgroup who either did not take drugs which can influence serum lipid levels or who had taken these drugs but the dose was not changed during the observation period. For a better understanding of the significance of these findings, studies with a larger number of patients are needed.

\section{References}

1. UK Prospective Diabetes Study (UKPDS) Group. Intensive blood-glucose control with sulphonylureas or insulin compared with conventional treatment and risk of com- plications in patients with type 2 diabetes (UKPDS 33). Lancet. 1998;352(9131):837-853.

2. Kahn SE, Haffner SM, Heise MA, Herman WH, Holman RR, Jones NP, Kravitz BG, et al. Glycemic durability of rosiglitazone, metformin, or glyburide monotherapy. $\mathrm{N}$ Engl J Med. 2006;355(23):2427-2443.

3. Foley JE, Sreenan S. Efficacy and safety comparison between the DPP-4 inhibitor vildagliptin and the sulfonylurea gliclazide after two years of monotherapy in drugnaive patients with type 2 diabetes. Horm Metab Res. 2009;41(12):905-909.

4. Seck T, Nauck M, Sheng D, Sunga S, Davies MJ, Stein PP, Kaufman KD, et al. Safety and efficacy of treatment with sitagliptin or glipizide in patients with type 2 diabetes inadequately controlled on metformin: a 2-year study. Int J Clin Pract. 2010;64(5):562-576.

5. Hollander PL, Li J, Frederich R, Allen E, Chen R, Investigators CV. Safety and efficacy of saxagliptin added to thiazolidinedione over 76 weeks in patients with type 2 diabetes mellitus. Diab Vasc Dis Res. 2011;8(2):125-135.

6. Rosenstock J, Niggli M, Maldonado-Lutomirsky M. Long-term 2-year safety and efficacy of vildagliptin compared with rosiglitazone in drug-naive patients with type 2 diabetes mellitus. Diabetes Obes Metab. 2009;11(6):571578.

7. Scirica BM, Bhatt DL, Braunwald E, Steg PG, Davidson J, Hirshberg B, Ohman P, et al. Saxagliptin and cardiovascular outcomes in patients with type 2 diabetes mellitus. N Engl J Med. 2013;369(14):1317-1326.

8. Del Prato S, Camisasca R, Wilson C, Fleck P. Durability of the efficacy and safety of alogliptin compared with glipizide in type 2 diabetes mellitus: a 2-year study. Diabetes Obes Metab. 2014;16(12):1239-1246.

9. Takebayashi K, Sakurai S, Suzuki T, Hori K, Terasawa T, Naruse R, Hara K, et al. Effect of combination therapy with alogliptin and lansoprazole on glycemic control in patients with type 2 diabetes. Endocr J. 2014;61(10):10311039.

10. Takebayashi K, Inukai T. Effect of proton pump inhibitors on glycemic control in patients with diabetes. World J Diabetes. 2015;6(10):1122-1131.

11. Takebayashi K, Terasawa T, Naruse R, Hara K, Suetsugu $\mathrm{M}$, Tsuchiya $\mathrm{T}$, Inukai $\mathrm{T}$. Long-term evaluation of glycemic control in patients with type 2 diabetes receiving either alogliptin and lansoprazole or alogliptin mono-therapy for 3 months followed by alogliptin mono-therapy: a retrospective analysis. Dokkyo J Med Sci. 2015;42(3):117-125.

12. Takebayashi K, Terasawa T, Naruse R, Hara K, Suetsugu $\mathrm{M}$, Tsuchiya $\mathrm{T}$, Inukai $\mathrm{T}$. The effect of switching from either mitiglinide or glimepiride to repaglinide on both glycemic control and oxidative stress in patients with type 2 diabetes. Dokkyo J Med Sci. 2016;43(1):5-13.

13. Yamazaki M, Hasegawa G, Majima S, Mitsuhashi K, Fukuda T, Iwase H, Kadono M, et al. Effect of repaglinide versus glimepiride on daily blood glucose variability and changes in blood inflammatory and oxidative stress markers. Diabetol Metab Syndr. 2014;6:54.

14. Matsuda T, Nishimura R, Sano H, Isaka T, Ebara F, 
Akutsu T, Someya Y, et al. Appropriate glimepiride dosage when switching from glibenclamide/gliclazide in type 2 diabetes a Japanese population. J Japan Diab Soc. 2005;48(3):159-164.(in Japanese).

15. Takebayashi K, Suetsugu M, Wakabayashi S, Aso Y, Inukai T. Retinol binding protein-4 levels and clinical features of type 2 diabetes patients. J Clin Endocrinol Metab. 2007;92(7):2712-2719.

16. Betteridge DJ. Effects of pioglitazone on lipid and lipoprotein metabolism. Diabetes Obes Metab. 2007;9(5):640647.

17. Mu J, Woods J, Zhou YP, Roy RS, Li Z, Zycband E, Feng Y, et al. Chronic inhibition of dipeptidyl peptidase-4 with a sitagliptin analog preserves pancreatic beta-cell mass and function in a rodent model of type 2 diabetes. Diabetes. 2006;55(6):1695-1704.

18. Kirino Y, Sei M, Kawazoe K, Minakuchi K, Sato Y. Plasma dipeptidyl peptidase 4 activity correlates with body mass index and the plasma adiponectin concentration in healthy young people. Endocr J. 2012;59(10):949-953.

19. Ayaori M, Iwakami N, Uto-Kondo H, Sato H, Sasaki M, Komatsu T, Iizuka M, et al. Dipeptidyl peptidase-4 inhibitors attenuate endothelial function as evaluated by flow-mediated vasodilatation in type 2 diabetic patients. $\mathrm{J}$ Am Heart Assoc. 2013;2(1):e003277.

20. Tahara N, Yamagishi SI, Bekki M, Kodama N, Nakamura T, Sugiyama Y, Oshige T, et al. Anagliptin, A dipeptidyl peptidase-4 inhibitor ameliorates arterial stiffness in association with reduction of remnant-like particle cholesterol and alanine transaminase levels in type 2 diabetic patients. Curr Vasc Pharmacol. 2016;14(6):552-562.

21. Kaku K, Itayasu T, Hiroi S, Hirayama M, Seino Y. Efficacy and safety of alogliptin added to pioglitazone in Japanese patients with type 2 diabetes: a randomized, double-blind, placebo-controlled trial with an openlabel long-term extension study. Diabetes Obes Metab. 2011;13(11):1028-1035.

22. Aoki K, Ijima T, Kamiyama H, Kamiko K, Terauchi Y. Anagliptin decreases serum lathosterol level in patients with type 2 diabetes: a pilot study. Expert Opin Pharmacother. 2015;16(12):1749-1754

23. Yano $\mathrm{W}$, Inoue $\mathrm{N}$, Ito $\mathrm{S}$, Itou $\mathrm{T}$, Yasumura $\mathrm{M}$, Yoshinaka $\mathrm{Y}$, Hagita S, et al. Mechanism of lipid-lowering action of the dipeptidyl peptidase-4 inhibitor, anagliptin, in lowdensity lipoprotein receptor-deficient mice. J Diabetes Investig. 2017;8(2):155-160. 